\title{
Analysis of the Influence of Probiotics on Intestinal Micro-ecology and Related Diseases
}

\author{
Luo YANG ${ }^{1}$, Hong GUO ${ }^{1, *}$, Yan-Ling SHEN ${ }^{2}$, Li LI $^{2}$, You-Hua LIU' ${ }^{1}$, Dan ZHAO', \\ Hai-Yan WANG ${ }^{1}$, Shao-Hua GONG ${ }^{1}$, Xiao-Ping $\mathrm{YI}^{1}$ \\ ${ }^{1}$ School of Nursing, Beijing University of Chinese Medicine, Beijing 100029, China;
}

${ }^{2}$ China-Japan Friendship Hospital, Beijing 100029, China

\begin{abstract}
Probiotics are a kind of living microorganisms added into food or drug, which are beneficial to our health. Probiotics can regulate the balance of microecosystem in intestine. Recently, people have paid more attention to the effect on the intestinal microecology. This article mainly made a review on the mechanism and application of probiotics.
\end{abstract}

Key words: Probiotics; Intestinal micro-ecosystem; Intestinal micro-ecology; Related diseases

\section{Introduction}

The intestinal micro-ecological system is the largest and most complex micro-ecological system in the human body. There are a large number of different types and different kinds of bacteria, including 300-500 kinds of bacteria, close to 2 million genotypes, and the number of bacteria in the intestines is about $10^{14}$ [1] The normal flora of the adult intestines are bifidobacteria, bacteroides, digestive cocci, bacillus, lactobacilli, clostridium, veillonococcus and buds. The intestinal flora is divided into three parts: (1) Physiological bacteria are obligate anaerobic bacteria which have symbiotic relationship with the host and are the dominant floras of the intestine, such as bifidobacteria, bacteroides, eubacteria and peptococcus ${ }^{[2]}$. The main constituents of the membrane flora have nutritional and immunomodulatory effects. (2) Conditional pathogen coexist with the host. They are mainly facultative anaerobic bacteria but are the non-dominant flora of the

\section{*Corresponding Author:}

Hong GUO, School of Nursing, Beijing University of Chinese Medicine, Beijing 100029, China E-mail: guohong2015@163.com.

Received:August 23 2019, Revised: September 10 2019,Accepted: September 20 2019, Online: November 152019 intestine, such as enterococcus, enterobacter. Conditional pathogens are harmless to the intestinal micro-ecological balance and are invasive under certain conditions and harmful to humans; (3) Pathogens: There are few opportunities for pathogens to colonize the human body for a long time. When the ecological balance is kept, these bacteria are small, and won't cause disease. If the amount exceeds the normal level, it can cause human diseases such as proteus, pseudomonas and clostridium ${ }^{[3]}$. From birth, the mechanism by which the human gut is tolerant to food and flora is gradually established. The specificity of the gastrointestinal mucosa is to maintain a delicate balance between normal immune response and immune tolerance, which involves down-regulation of mucosal immunity ${ }^{[1]}$.

Under normal circumstances, the ecosystem consisting of normal flora, host and external environment is balanced. However, due to changes in living environment, age, physiological state, mental stimulation, or due to abuse of antibiotics, exposure to radiation, etc., intestinal microecology may be destroyed, causing micro-ecological 
disorders, such as bacterial flora imbalance, bacterial imbalance and bacterial colonization transfer, resulting in constipation, acute and chronic diarrhea, stomach enteritis, intestinal dysfunction, and diseases such as foreign infections. Long-term dysbacteriosis can also cause the host to produce various diseases or promote aging and even cause cancer ${ }^{[4]}$. It is worth mentioning that in recent years, the problem of antibiotic resistance has caused widespread concern. The adverse consequences caused by the abuse of antibiotics have brought many difficulties to the treatment of certain diseases. Probiotics can pass non-immune regulation. Stabilizing the micro-ecological environment can also enhance the body's immune defense function by improving the body's specific immunity.

\section{Mechanism of Action of Probiotics}

\section{Colonization antagonism of probiotics}

The pathogenic bacteria bind to the intestinal mucosal surface receptor and adhere to the intestinal mucosa. This is a prerequisite for its colonization and clinical symptoms ${ }^{[2-3]}$. Many probiotics prevent the colonization of pathogenic bacteria by competing with them for the same complex carbohydrate receptors on lipids and proteins on the intestinal epithelial microvilli. Some scholars ${ }^{[4]}$ applied the human intestinal epithelial cell line $\mathrm{CaCo}-2$ cells for the adhesion assay of various bacteria in vitro, which proved that different probiotics have different adhesion modes. L.gasseri adheres through sugars and glycoproteins, and its adhesion is the strongest. Lactobacillus acidophilus relies on sugars and divalent calcium ions to adhere. Bifidobacteria adheres to intestinal epithelial cells through lipid wall phosphate, forming a membrane barrier, and producing extra-cellular glycosidases, which are degraded on epithelial cells. The complex polysaccharides of potential pathogens and their endotoxin-binding receptors competitively inhibit endogenous and exogenous potential pathogenic bacteria in the intestines, adhere to and colonize intestinal epithelial cells, and cause them to leave the infected intestines to colonization antagonism.

\section{Probiotics inhibit the pathogenic bacteria}

Probiotics can inhibit the colonization and proliferation of pathogenic bacteria. In addition to the quantitative advantages, it can also inhibit the growth and reproduction of pathogenic bacteria by secreting antibacterial substances ${ }^{[5]}$. Bifidobacteria and lactic acid bacteria can ferment sugars to produce acetic acid and lactic acid in the body, so that the intestines are in an acidic environment, forming a protective barrier for intestinal tract, protecting the microenvironment in the intestinal tract, preventing the invasion, adhesion and colonization of pathogenic bacteria. And it inhibits the growth of abnormal flora such as shigella, salmonella typhi, proteus, pseudomonas aeruginosa and fungi and the production of harmful metabolites.

Certain probiotics produce substances with a broad spectrum of antibacterial effects, such as bacteriocin, hydrogen peroxide, lipophilic molecules, diacetyl, carbon dioxide, acetaldehyde, etc., which are responsible for escherichia coli, salmonella, streptococcus in the intestine. All have antibacterial or bactericidal effects. Different antibacterial substances have different effects on Grampositive and Gram-negative bacteria; some antibacterial substances can also reduce the production of harmful substances such as ammonia and amines in the intestine, reduce endotoxin, and improve intestinal function.

In addition, probiotics can also prevent pathogenic bacteria from invading intestinal mucosal cells by producing extracellular glycosidases that degrade the complex polysaccharides of pathogenic bacteria and endotoxin-binding receptors. Falk et $a l^{[6]}$ found that bifidobacteria can produce extracellular glycosidases, which can degrade intestinal mucin oligosaccharides and lactic acid series type I glycosphingolipids to prevent bacterial invasion.

\section{The nutritional role of probiotics}

Probiotics can directly produce a variety of nutrients such as vitamins, amino acids, short-chain fatty acids, growthpromoting factors, etc., and participate in the metabolism of animals. Some microorganisms can synthesize riboflavin, pantothenic acid, folic acid, B vitamins, etc. when they grow and reproduce in animals, and participate in some important metabolic reactions of the body. 
Probiotics can also supplement the host's deficiency in digestive enzymes by using certain enzymes (such as galactosidase), which help to decompose the nutrients that are not fully hydrolyzed and absorbed by the upper digestive tract, so as to help the host to further absorb and utilize nutrient substances, such as vitamins, amino acids, trace elements and certain inorganic salts (such as calcium, phosphorus, iron, cobalt, etc.) necessary for the body.

\section{The Effect of Probiotics on the Disease}

\section{Application of probiotics in infectious diarrhea}

In 1999, the Law on the Prevention and Control of Infectious Diseases in China referred to diarrhea caused by microorganisms other than cholera, dysentery, typhoid and paratyphoid as "infectious diarrhea" and classified as a Class $\mathrm{C}$ infectious disease. At present, the incidence of infectious diarrhea in China is the highest among all infectious diseases.

Pathogenesis of infectious diarrhea: When the ratio of pathogenic bacteria to normal flora in the intestine is imbalanced, the pathogenic bacteria in the intestine increase, and the secreted enterotoxin increases; the increased pathogenic bacteria can also invade and damage the intestinal epithelial cells; some overgrowth bacteria can affect the intestines. The energy metabolism of epithelial cells leads to damage of epithelial cells and induces intestinal inflammation ${ }^{[7]}$; intestinal flora can increase intestinal permeability, impaired barrier function, intestinal antigen, endotoxin, etc. Inflammatory substances enter the lamina propria of the intestinal mucosa, causing aggregation of macrophages; the pathogens are rich in intestinal mucosa, causing absorption disorders, which can cause absorbent diarrhea and osmotic diarrhea.

The role of probiotics in the treatment of diarrhea: The live bacterial preparation has an action of producing active substances such as organic acid, bacteriocin, or hydrogen peroxide, and can inhibit or kill the pathogenic bacteria. Double-clicking on physiological bacteria such as bacillus, lactobacillus, and enterobacter faecium can produce short-chain fatty acids, which lower the intestinal $\mathrm{pH}$ and antagonize pathogenic bacteria. Lactobacillus casei GG, lactobacillus plantarum, bacillus subtilis and enterococcus can produce substances such as benzoic acid, which can antagonize gram-positive and gram-negative pathogens, and can produce sterilized hydrogen peroxide. Bifidobacteria can produce bile acid decomposed into free bile acids in the intestine, which in turn has a strong inhibitory effect on saprophytic bacteria. The live bacterial preparation can be colonized between intestinal epithelial cells to form a biofilm-like structure, which hinders the occupying and reproduction of the invading bacteria. Bifidobacteria can interact with intestinal mucosal epithelial cells through the production of lipoteichoic acid, and tightly bind together with other anaerobic bacteria to occupy the surface of the intestinal mucosa and form a biological barrier. The physiological live bacterial preparation competes with the disease-causing microorganisms for the adhesion site in the intestinal mucosa, thereby occupying the space, participating in the construction of the intestinal biological barrier, competing or rejecting the colonization of the pathogenic bacteria and the invasive bacteria ${ }^{[8]}$. The living bacteria in the active preparation can promote the production of antibodies by human B cells, and also activate immune cells as nonspecific immunoregulators to promote the development and maturation of the immune system. Experimental studies ${ }^{[9-11]}$ have shown that bifidobacteria, lactobacillus and other complex live bacterial preparations can increase the proportion and activity of lymphocytes and NK cells in the blood, stimulate the production of antibodies by macrophages, help kill pathogenic microorganisms, and improve production of specific antibodies. Probiotics are beneficial to restore the ecological balance of intestinal flora, help control diarrhea, increase the number of probiotics, inhibit the growth and reproduction of intestinal pathogenic bacteria, and limit the excessive growth of pathogenic bacteria. The application of microecological preparations for the treatment of infectious diarrhea, especially viral diarrhea, can significantly shorten the course of treatment ${ }^{[12]}$. Study ${ }^{[13]}$ has shown that bifidobacteria, bifidobacteria and lactic acid bacteria are safe and effective in the treatment of pediatric infectious diarrhea. And multiple probiotics are 
more effective than single probiotics. In addition, patients with chronic persistent diarrhea who have developed dysbacteriosis are in great need of micro-ecological live bacterial preparations ${ }^{[14]}$.

\section{The role of probiotics in inflammatory bowel disease}

Inflammatory bowel disease (IBD) is a group of chronic non-specific intestinal inflammatory diseases whose causes are not well defined, including Crohn's disease (CD) and ulcerative colitis (UC).

Pathogenesis of inflammatory bowel disease: At present, most scholars believe that its pathogenesis is closely related to various factors such as immunity, genetic susceptibility, intestinal flora and environment. To date, 163 genetic polymorphisms have been found to be associated with the onset of UC or CD. Most of those involve the regulation of the immune response of the intestine to the flora and the maintenance of the integrity of the intestinal epithelial barrier. The disorder of intestinal immunity and bacterial interaction between individuals with genetic predisposition is an important pathogenesis of IBD. Intestinal flora imbalance and impaired intestinal mucosal barrier function are characteristics of IBD onset $^{[15]}$. The intestinal flora plays an important role in the pathogenesis of IBD and throughout the pathogenesis. Therefore, maintaining the intestinal flora stability and protecting the intestinal mucosal barrier integrity have become the key to prevention and treatment of IBD and the focus of scholars in various countries.

The role of probiotics in the treatment of IBD: The mechanism of probiotics on the treatment of IBD is not fully understood. The current research suggests that the following aspects may be involved: probiotics in the colon compete with pathogenic microorganisms for nutrients and produce antibacterials to effectively prevent the invasion and reproduction of pathogenic microorganisms. By fermenting undigested carbohydrates, probiotics can synthesize energy for colonic mucosal epithelium - shortchain fatty acids. It also regulates cellular and humoral immunity, especially intestinal mucosa-associated lymphoid tissue $(\mathrm{GALT})^{[16]}$, regulate intestinal wall cell gene expression and cell differentiation, repair damaged intestinal mucosal epithelial tissue, reduce intestinal $\mathrm{pH}$, inhibit the growth of pathogenic bacteria, stimulate the growth of beneficial bacteria, especially bifidobacteria, lactic acid bacteria ${ }^{[17]}$. Bacteria adhere to intestinal epithelial cells to prevent pathogenic bacteria from crossing the intestinal mucosal epithelial barrier, thus preventing bacterial translocation, increase the surface area of the small intestine villi, promote the absorption of nutrients, and produce other chemicals, such as neurotransmitters normally present in the intestine and regulate intestinal sensation and movement.

\section{Application of probiotics in the treatment of irritable bowel syndrome}

Irritable bowel syndrome (IBS) is a common non-organic gastrointestinal dysfunction with abdominal pain or abdominal discomfort as the main clinical symptoms. The disease can be improved after defecation, often accompanied by changes in bowel habits, the mechanism is complex, and the clinical manifestations are diverse.

Pathogenesis of IBS: The pathogenesis of IBS is complex and has not yet been fully elucidated. Most studies suggest that it is associated with multiple factors such as intestinal motility abnormalities, inflammation, intestinal flora disorder, and genetic factors ${ }^{[18-21]}$. Studies have shown that patients with IBS have intestinal flora imbalance ${ }^{[22]}$ and intestinal barrier function abnormalities $^{[23]}$. There is currently no monotherapy or a drug that is completely effective for IBS $^{[24]}$, but the combination of administration methods can alleviate the symptoms of IBS to some extent. This study combined probiotics preparations and glutamine granules in the treatment of diarrhea-type IBS, the results show that it can regulate intestinal microflora to maintain intestinal microecological balance, protect intestinal mucosa, and relieve diarrhea-type irritable bowel symptoms of patients with syndrome.

Therapeutic effect of probiotics in IBS: Patients with diarrhea-type IBD have obvious intestinal flora imbalance and intestinal mucosal barrier dysfunction, while probiotic preparations can increase enteric probiotics such as 
lactobacillus enterica, and reduce intestinal pathogens and pathogenic bacteria. To maintain intestinal microecological balance, combined with glutamine can enhance immunity, reduce inflammation, protect intestinal mucosa, promote the relief of abdominal pain, diarrhea and other symptoms, so it is effective for IBD, and easy to take, with less adverse reactions, and worth promoting.

\section{The role of probiotics in cirrhosis}

Studies have shown that probiotics, especially lactobacillus, enhance host immunity and fight infection by enhancing phagocytic activity and increasing $\operatorname{IgA}$ secretion, and enhancing cellular immunity by increasing the number of natural killer cells and $\mathrm{T}$ lymphocytes ${ }^{[25]}$. Wiest et al. ${ }^{[26]}$ pointed out that Lactobacillus can correct intestinal bacterial overgrowth, stabilize intestinal mucosal barrier, improve local defense function, thereby reducing the occurrence of BT and spontaneous bacterial peritonitis. In a doubleblind randomized trial, Lata et al. ${ }^{[27]}$ randomized 39 patients diagnosed with cirrhosis and treated them with E.coli Nissle and placebo for 42 days. The results showed that the E.coli Nissle group could significantly improved intestinal flora imbalance $(P<0.001)$, effectively reduce endotoxemia and improve liver function. Cesaro et al. ${ }^{[28]}$ believe that probiotics are used in patients with cirrhosis, even if only a small dose, the effect is worthy of recognition.

\section{The role of probiotics in the treatment of cancer}

A large number of in vitro and in vivo experiments have proved that probiotics have a positive effect on cancer prevention and postoperative sensory control. The antitumor effect of probiotics has been validated in many animal experiments. Probiotics prevent colon cancer ${ }^{[29]}$, in which mechanism NF- $\mathrm{BB}$ signaling is activated in host cells by binding lipopolysaccharide, peptidoglycan, and CpG-DNA in probiotics to Toll-like receptors (TLRs) on the surface of host intestinal mucosal cells. The conduction pathway is related. It can also be used for colorectal cancer surgery, to improve intestinal barrier function, reduce the occurrence of bacterial translocation, and it is conducive to the recovery of early postoperative inflammatory response; probiotics also have a positive effect on the treatment of gastric tumors, Lactobacillus rhamnosus (LGG) homogenate can reduce ODC mRNA The content and activity, and can increase the content and activity of SSAT mRNA, thereby reducing the content of polyamines and tumor expansion, so LGG can be used as an alternative to prevent gastric tumors, and can overcome the side effects of therapeutic drugs. In the aspect of liver tumors, oral probiotics can reduce the incidence of postoperative complications in patients with liver cancer, and have certain clinical application value. Study ${ }^{[30]}$ has shown that, probiotics can block the intake of aflatoxin in the intestine. This reduces the probability of liver cancer growth through the aflatoxin community in food; several studies ${ }^{[31-33]}$ have shown that probiotics can induce apoptosis and therefore have a certain inspiration for the treatment of breast cancer and leukemia.

\section{Summary}

Numerous experimental studies have shown that the intake of probiotics has a certain regulatory effect on the human intestinal flora. However, many mechanisms of action are not well understood, and the use of the dose is not yet clear, so further research is needed. Although probiotics have many benefits, they are not the so-called "golden oil". Only reasonable development and utilization, strict market monitoring and screening, can probiotics make truly "benefit for life".

\section{Declaration}

All authors of this article declare no conflict of interest.

\section{Acknowledgments}

The authors gratefully acknowledge the authors who list in those literatures which were cited in the present review.

\section{References}

1 Sun CY, Tang QY. Therapeutic effects of intestinal microecology and inflammatory bowel disease and probiotics. J Clin Digest Dis, 2014, 26(5): 312-314.

2 Zhang DY, Ji HF, Xu YL. Research progress on the effects of probiotics on intestinal microbial ecology in 
animals. Chin Anim Husb Vet Med, 2007, 34(3): 1518.

3 Cousin FJ, Mater DDG, Foligné B, et al. Progress in Probiotic Propionate. Dairy Sci Tech, 2011, 91(1): $1-26$.

4 Fernández MF, Boris S, Barbés C. Probiotic properties of human lactobacilli strains to be used in the gastrointestinal tract. J Applied Microbiol, 2003, 94(3): 449-455.

5 Cheng W, Liu ZY. Experimental Study on Prevention and Control of Salmonella Typhimurium Infection by Bifidobacterium. Chin J Microecol, 1999, 11(5): 260263.

6 Larson G, Falk P, Hynsjö L, et al. Faecal Excretion of Glycosphingolipids of Breast-fed and Formula-fed Infants. Microb Ecol Health Dis, 2009, 3(6): 305-319.

7 Huang YK, Yang MF, Li HL. Advances in research on changes of gastrointestinal flora in children with common gastrointestinal diseases. Chin J Pract Pediatr, 2007, 22(7): 481-484.

8 Gibson GR, Probert HM, Loo JV, et al. Dietary modulation of the human colonic microbiota: updating the concept of prebiotics. J Nutr, 2004, 17(2): 259-275.

9 Li YQ. Progress in Physiology and Pharmacology of Bifidobacteria. Mod Applied Pharm, 1995, (6): 11-12.

10 Zhu JC, Li SM, Wei XQ. Physiological Effects and Research Progress of Bifidobacteria on Human Body. Chin J Microecol, 2000, 12(1): 53-55.

11 Xiong DX. Modern Intestinal Microecology. Beijing: China Science and Technology Press. 2003, 189-212.

12 Nie QH. Current status of research on infectious diarrhea. Chin J Pract Intern Med, 2003, 23(10): 631633.

$13 \mathrm{Xu}$ WM, Yang W, Wu D, et al. Clinical study of microecological preparations in the treatment of infectious diarrhea in children. J Nantong Univ (Medical Sciences), 2007, 27(2): 132-133.

14 Dong ZQ, Fang HS. Minutes of the International Symposium on Diarrhea in China and Children's Diarrhea. J Clin Pediatr, 2004, (6): 414-415.

15 Viladomiu M, Hontecillas R, Yuan L, et al. Nutritional protective mechanisms against gut inflammation. J Nutr Biochem, 2013, 24(6): 929-939.
16 Brownawell AM, Caers W, Gibson GR, et al. Prebiotics and the Health Benefits of Fiber: Current Regulatory Status, Future Research, and Goals. J Nutr, 2012, 142(5): 962-974.

17 Sarowska J, Choroszykról I, Regulskailow B, et al. The therapeutic effect of probiotic bacteria on gastrointestinal diseases. Adv Clin Exper Med, 2013, 22(5): 759-766.

18 Qin JJ, Bai PP. Therapeutic effect of mechangan and trimebutine maleate on diarrhea-predominant irritable bowel syndrome. Chin Med Guide, 2009, 7(4): 18-19.

19 Zhang XN, Jin SL, Liu BZ, et al. Effects of intervention of intestinal flora on the symptomatic syndrome of irritable bowel syndrome. Chin Med, 2012, 7(12): 1547-1549.

$20 \mathrm{Lu} \mathrm{XF}, \mathrm{Yu}$ GC. Research progress of resveratrol on irritable bowel syndrome. J Pract Med, 2013, 29(18): 3088-3090.

21 Wang LY, Yang YS, Cao XP, et al. Epidemiological investigation of functional bowel disease in rural areas of Lankao County, Henan Province. Chin J Clin Med, 2012, 19(5): 485-487.

22 Jiang YF, Lao SX, Qi ZY, et al. Changes of intestinal flora in spleen-stomach damp-heat syndrome of diarrhea-type irritable bowel syndrome. Chin J Integr Trad Western Med, 2006, 26(3): 218-220.

23 Zeng J. Study on intestinal mucosal barrier function of irritable bowel syndrome. Jinan: Shandong University, 2008.

$24 \mathrm{Hu}$ PJ. Treatment of standardized irritable bowel syndrome. J Clin Digest Dis, 2008, 20(5): 259-260.

25 Guerrero HI, Torre DA, Vargas VF, et al. Intestinal flora, probiotics, and cirrhosis. Ann Hepatol, 2008, $7(2): 120$.

26 Dong D, Yin L, Qi Y, et al. Protective Effect of the Total Saponins from Rosa laevigata Michx Fruit against Carbon Tetrachloride-Induced Liver Fibrosis in Rats. Nutrients, 2015, 7(6): 4829.

27 Lata J, Novotný I, Príbramská V, et al. The effect of probiotics on gut flora, level of endotoxin and ChildPugh score in cirrhotic patients: results of a doubleblind randomized study. Eur J Gastroenterol Hepatol, 2007, 19(12): 1111-1113.

28 Cesaro C, Tiso A, Del PA, et al. Gut microbiota and probiotics in chronic liver diseases. Dig Liver Dis, 
2011, 43(6): 431-438.

29 Wollowski I, Rechkemmer G, Poolzobel B L. Protective role of probiotics and prebiotics in colon cancer. Am J Clin Nutr, 2001, 73(Suppl 2): 451.

30 Russo F, Refolo MG, Orlando A, et al. Lactobacillus rhamnosus GG Influences Polyamine Metabolism in HGC-27 Gastric Cancer Cell Line: A Strategy Toward Nutritional Approach to Chemoprevention of Gastric Cancer. Curr Pharm Des, 2010, 16(7): 847-853.

31 Esfarjani SV, Ahvazi NC, Doberjovi M, et al. Compa- rative study of some breast cancer risk factors in patients with breast cancer and leukemia in Ahvaz Shafa Hospital. Sci Med J, 2010, 35(1): 159.

32 Cornetta K, Berebitsky D, Behnia M, et al. A retroviral vector expressing human interferon gamma upregulates MHC antigen expression in human breast cancer and leukemia cell lines. Cancer Gene Ther, 1994, 1(2): 91-98.

33 Hudis C. Breast Cancer and Leukemia: The forest for the trees? Ann Surg Oncol, 2002, 9(8): 717-718. 